\title{
Leishmaniose visceral em Sobral, Ceará: análise epidemiológica comparativa de dois quinquênios
}

\author{
Visceral leishmaniasis in Sobral, Ceará: comparative epidemiological analysis of two five- \\ year periods
}

\section{Leishmaniasis visceral en Sobral, Ceará: análisis epidemiológico comparativo de dos quinquenios}

Walter Oliveira Rios Junior ${ }^{1 *}$, José Francisco Igor Siqueira Ferreira ${ }^{1}$, Victor Carneiro de Souza1 ${ }^{1}$ José Lucas Martins Costa', Ulli Aguiar Vasconcelos², Madelline Alves Vasconcelos², Lucas Lima Ellery², Antonio Henrique Barroso do Vale Filho², Maria Mariana Rocha Gomes², Valéria Fontenele Marques².

\section{RESUMO}

Objetivo: Realizar uma análise epidemiológica comparativa dos casos de Leishmaniose Visceral (LV) no município de Sobral. Métodos: Trata-se de um estudo descritivo, documental e de caráter quantitativo. Foram utilizados dados, referentes aos períodos de 2009 a 2013 e de 2014 a 2018, fornecidos pelo Ministério da Saúde (MS), através do Sistema de Informação de Agravos de Notificação (SINAN). Os dados de cada variável foram analisados por meio da estatística descritiva, através do cálculo da média, do desvio padrão e de percentis, por ano de ocorrência. Por fim, os resultados de cada quinquênio analisado foram comparados entre si. Resultados: Houve uma queda de $19,59 \%$ na taxa incidência, de $28,52 \%$ na taxa de mortalidade e de $11,42 \%$ no Coeficiente de Letalidade de LV. O predomínio da doença no sexo masculino, na faixa etária pediátrica, em indivíduos pardos e de baixa escolaridade se manteve. Além disso, houve uma inversão do fenômeno da urbanização. Conclusão: A redução das taxas de incidência, mortalidade e letalidade da doença no município refletem uma melhora do sistema de saúde, da prevenção ao diagnóstico e tratamento. No entanto, tais taxas ainda permanecem elevadas quando comparada com outras regiões e o caráter de endemia da doença no município ainda se mantém.

Palavras-chave: Leishmaniose visceral, Sobral, Epidemiologia.

\begin{abstract}
Objective: To execute a comparative epidemiological analysis of cases of Visceral Leishmaniasis $(\mathrm{VL})$ in the municipality of Sobral. Methods: This is a descriptive, documentary and quantitative study. Data provided, referring to the periods from 2009 to 2013 and from 2014 to 2018, by the Ministry of Health, through the Information System of Notifiable Diseases, were used. The data of each variable were analyzed using descriptive statistics, by calculating the mean, standard deviation and percentiles, by year of occurrence. Finally, the results of each five-year period analyzed were compared. Results: There was a $19.59 \%$ decrease in the incidence rate, $28.52 \%$ in the mortality rate and $11.42 \%$ in the VL lethality coefficient. The prevalence of the disease in males, in the pediatric age group, in brown individuals and those with low schooling remained. In addition, there was a reversal of the urbanization phenomenon. Conclusion: The reduction in the incidence, mortality and lethality rates of the disease in the municipality reflects an improvement in the health system, from prevention to diagnosis and treatment. However, these rates still remain high when compared to other regions and the endemic nature of the disease in the municipality is still maintained.
\end{abstract}

Keywords: Visceral leishmaniasis, Sobral, Epidemiology.

${ }^{1}$ Universidade Federal do Ceará (UFC), Sobral - CE. *E-mail: walteroriosjr@gmail.com

${ }^{2}$ Centro Universitário INTA (UNINTA), Sobral - CE. 


\section{RESUMEN}

Objetivo: Realizar un análisis epidemiológico comparativo de los casos de Leishmaniasis Visceral (LV) en el municipio de Sobral. Métodos: Se trata de un estudio descriptivo, documental y de carácter cuantitativo. Se utilizaron datos, referido a los periodos de 2009 a 2013 y de 2014 a 2018, proporcionados por el Ministerio de Salud a través del Sistema de Información de Agravios de Notificación. Los datos de cada variable se analizaron mediante la estadística descriptiva, calculando la media, la desviación estándar y los percentiles por año de aparición. Finalmente, los resultados de cada quinquenio analizado fueron comparados entre sí. Resultados: Hubo una caída de $19,59 \%$ en la tasa de incidencia, de $28,52 \%$ en la tasa de mortalidad y de $11,42 \%$ en el coeficiente de letalidad de LV. La prevalencia de la enfermedad entre los varones, en el grupo de edad pediátrica, entre los pardos y los de baja escolaridad se ha mantenido. Además, hubo una inversión del fenómeno de la urbanización. Conclusión: La reducción de las tasas de incidencia, mortalidad y letalidad de la enfermedad en el municipio reflejan una mejora del sistema de salud, de la prevención al diagnóstico y tratamiento. Sin embargo, estas tasas siguen siendo elevadas en comparación con otras regiones y el carácter de endemia de la enfermedad en el municipio todavía se mantiene.

Palabras clave: Leishmaniasis visceral, Sobral, Epidemiología.

\section{INTRODUÇÃO}

A Leishmaniose Visceral (LV), popularmente conhecida como "calazar", é uma doença sistêmica grave, causada por protozoários tripanosomatídeos do gênero Leishmania, parasita intracelular obrigatório das células do sistema fagocítico mononuclear, com uma forma flagelada ou promastigota, encontrada no tubo digestivo do inseto vetor e outra aflagelada ou amastigota nos tecidos dos vertebrados.

No Brasil, a espécie Leishmania chagasi é o principal agente etiológico da doença (MINISTÉRIO DA SAÚDE, 2020). A doença é transmitida para o homem através da picada da fêmea de insetos denominados flebotomíneos, popularmente conhecidos como "mosquito palha". No Brasil, a espécie Lutzomyia longipalpis é o principal vetor (MINISTÉRIO DA SAÚDE, 2020).

A incidência mundial estimada da LV é de 50.000 a 90.000 casos/ano, com mais de $95 \%$ dos novos casos ocorrendo em 10 países: Brasil, China, Etiópia, Índia, Iraque, Quênia, Nepal, Somália, Sudão do Sul e Sudão. No ano de 2018, 97\% dos casos do continente americano ocorreram no Brasil (WHO, 2020). As manifestações clínicas são caracterizadas por episódios irregulares de febre, perda de peso, hepatoesplenomegalia e anemia. Quando não tratada, a evolução é fatal em mais de 95\% dos casos (WHO, 2020).

Nas Américas, a LV é endêmica em 12 países. Entre 2001 e 2017 foram registrados 59.769 novos casos, resultando em uma média de 3.516 casos por ano. Nesse período, cerca de $96 \%$ (57.582) dos casos foram reportados pelo Brasil. (OPAS, 2019). No Brasil, a região nordeste é a mais acometida pela doença. No período de 2010 a 2017 foram confirmados 16.063 casos de LV em indivíduos residentes nessa região, com o maior número dos casos registrados nos estados do Ceará, Maranhão, Bahia e Piauí (LUCENA RV, MEDEIROS JS, 2018)

Segundo o Boletim Epidemiológico da Secretaria de Saúde do Estado do Ceará, a incidência e a letalidade da LV no estado vêm diminuindo. Entre 2008 e 2018, a média anual de casos confirmados foi de 578 e a incidência de 6,7 casos/ 100.000 hab. Os municípios com maior número de ocorrências de LV foram Fortaleza e Sobral (CEARÁ, 2018).

Dessa forma, este estudo tem o objetivo de realizar uma análise epidemiológica comparativa dos casos de Leishmaniose Visceral no município de Sobral, nos períodos de 2009 a 2013 e 2014 a 2018, por meio dos dados contidos no Sistema de Informação de Agravos de Notificação (SINAN) do Ministério da Saúde.

\section{MÉTODOS}

Trata-se de um estudo descritivo, documental e de caráter quantitativo. Foram utilizados dados fornecidos pelo MS através do Sistema de Informação de Agravos de Notificação (SINAN) para a realização de um estudo epidemiológico abordando todos os casos de Leishmaniose Visceral no município de Sobral, Ceará, 
notificados para o período de janeiro de 2009 a dezembro de 2013 (Período 1) e janeiro de 2014 a dezembro de 2018 (Período 2).

Os dados de cada ano foram atualizados e revisados nos meses de agosto do ano seguinte, segundo o SINAN. Através desses dados, o perfil epidemiológico da Leishmaniose Visceral e sua evolução foram delineados em cada período. Além disso, foram realizados comparativos entre os dois períodos analisados. Para o cálculo das taxas de incidência e de mortalidade, foram usadas as projeções anuais para a população, de acordo com o Instituto Brasileiro de Geografia e Estatística (IBGE), disponíveis para download no site do instituto (IBGE, 2020).

O cenário do estudo é o município de Sobral. O município está situado na região Noroeste do Ceará, a 235 quilômetros de Fortaleza. Limita-se ao norte com os municípios de Massapê, Santana do Acaraú e a Serra da Meruoca, ao sul com Santa Quitéria, Groaíras e Cariré, ao leste com Itapipoca, Irauçuba e Canindé, e ao oeste com Coreaú, Mucambo e Alcântara. Sobral é o segundo município mais desenvolvido do estado do Ceará e possui clima tropical, quente e seco com temperatura média de $30^{\circ} \mathrm{C}$ (OLIVEIRA LS, et al., 2013).

As variáveis obtidas para a análise epidemiológica foram: zona de residência do paciente acometido pela Leishmaniose Visceral, sua escolaridade, raça, sexo, presença de coinfecção HIV, gestação, realização de diagnósticos parasitários e/ou imunológicos, o critério de diagnóstico da doença e a evolução dos casos desses pacientes, de forma a traçar o perfil epidemiológico nos períodos analisados.

Os dados obtidos foram tabulados através do software Microsoft Office Excel e analisados por meio da estatística descritiva, através do cálculo da média (medida de tendência central), do desvio padrão (medida de dispersão) e de percentis, nos dados de cada variável, por ano de ocorrência, bem como os dados referentes a todo o quinquênio analisado. Os dados coletados são expressos como média \pm erro padrão da média e/ou em porcentagem.

\section{RESULTADOS E DISCUSSÃO}

Foi notificado em todo o município de Sobral, no período de 2009 a 2013, um total de 414 casos, com uma média anual de $82,8 \pm 17,85$ casos, o que corresponde a $15,22 \%$ dos casos do Estado do Ceará, com uma incidência média anual de 43,55 $\pm 9,78$ casos/100 mil habitantes. Já no período de 2014 a 2018, foram notificados 355 casos, com uma média anual de $71 \pm 23,71$ casos, o que corresponde a $15,63 \%$ dos casos estaduais, com uma incidência média anual de 35,02 $\pm 12,19$ casos/100 mil habitantes.

Em ambos os períodos, o valor encontrado para a incidência da doença no município é bem superior a incidência média no estado do Ceará de $6,35 \pm 1,26$ casos/100 mil habitantes e 5,08 $\pm 1,27$ casos/100 mil habitantes, respectivamente; e no Brasil de 1,9 \pm 0,19 casos/100 mil habitantes e 1,86 $\pm 0,18$ casos/100 mil habitantes, respectivamente (Gráfico 1).

Gráfico 1 - Incidência de Leishmaniose Visceral em Sobral, no Ceará e no Brasil, no período de 2009 a 2018 (em casos por 100 mil habitantes).

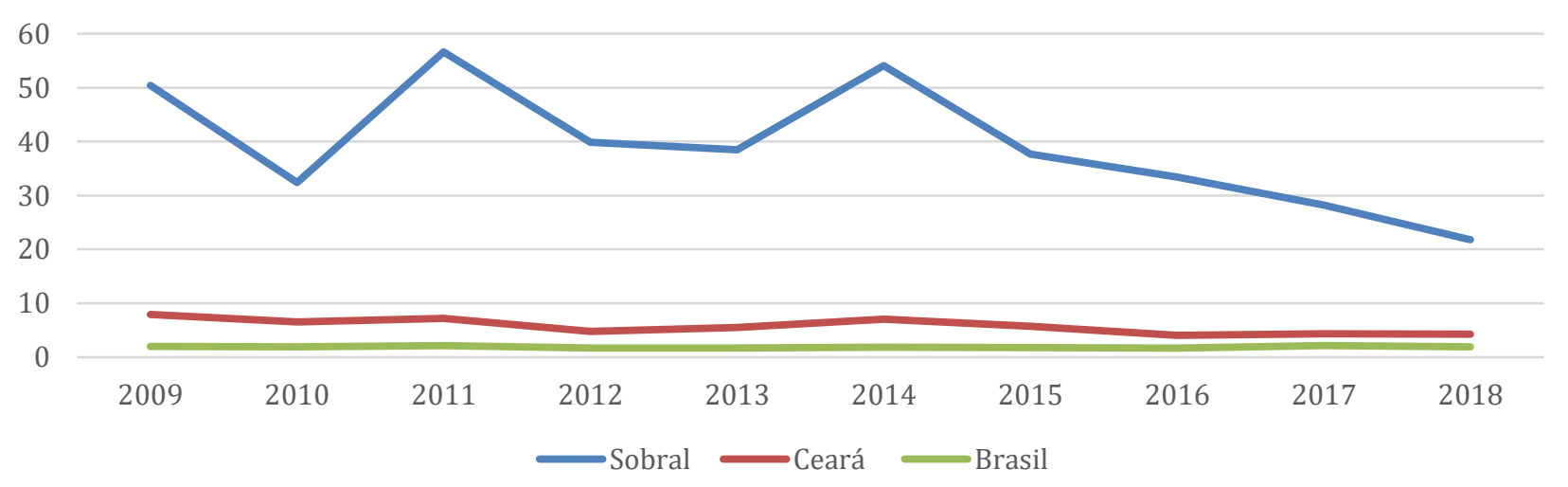

Fonte: Rios Junior WO, et al., 2020. Dados extraídos do Sistema de Informação de Agravos de Notificação (SINAN). 
Observou-se, portanto, uma queda de $19,59 \%$ na taxa de incidência de casos de LV, entre os períodos analisados. Tal fato demonstra uma efetividade nas medidas de prevenção da doença, como o combate ao mosquito vetor, empregadas no município. No entanto, mesmo com a queda importante da incidência nos últimos anos, o município mantém o caráter endêmico da Leishmaniose Visceral, com valores mais expressivos que aqueles evidenciados no estado do Ceará e no Brasil como um todo.

Diversos são os fatores que permitem a permanência da Leishmaniose Visceral como um problema em diversas regiões do país. Conforme o estudo realizado por Werneck GL (2016), um dos grandes problemas é a limitação de recursos humanos e financeiros no desenvolvimento e aplicação de estratégias de prevenção, o que contribui para a descontinuidade e/ou baixa cobertura das ações de controle. Além disso, a resistência social a permitir o acesso às residências pelas equipes está cada vez maior, principalmente no que se refere à eutanásia canina.

Justificativa semelhante foi evidenciada no estudo de Costa DNCC, et al. (2018). Na qual as medidas de controle do vetor (borrifação de inseticida e controle do reservatório canino), limitadas pela recusa à entrada no domicílio, apresentaram baixas coberturas no período estudado. Além da importante contribuição da falta de recursos materiais e humanos para esse resultado.

Em relação à zona de residência dos pacientes acometidos pela doença, de 2009 a 2013, 118 residiam em zona rural, com média de $23,6 \pm 8$ casos por ano, correspondendo a $29,06 \%$ de todas as notificações em Sobral. Já na zona urbana, 285 casos foram notificados, com média anual de $57 \pm 11$ casos, o que corresponde a $70,2 \%$ dos casos do município (Gráfico 2). Por fim, apenas 3 casos $(0,7 \%)$ foram registrados na zona periurbana. Já no quinquênio seguinte, $47,7 \%$ dos casos (166 casos, com média anual de $33,2 \pm 13$ ) notificados eram residentes da zona urbana e $52,3 \%$, da zona rural (182 casos, com média anual de $36,4 \pm$ 12,8).

Diferente do observado no estudo de Cavalcante IJM e Vale MR (2014), ocorreu, nos últimos 5 anos, uma inversão do cenário de acometimento da doença. No ano de 2014, o número de casos de Leishmaniose Visceral no município de Sobral se tornou mais prevalente na zona rural, em comparação com a zona urbana. Após esse ano, os números de casos se tornaram cada mais próximos, sem predomínio claro de incidência em uma zona de residência (Gráfico 2).

Gráfico 2 - Número de casos de Leishmaniose Visceral em Sobral, por zona de residência, no período de 2009 a 2018 (números absolutos).

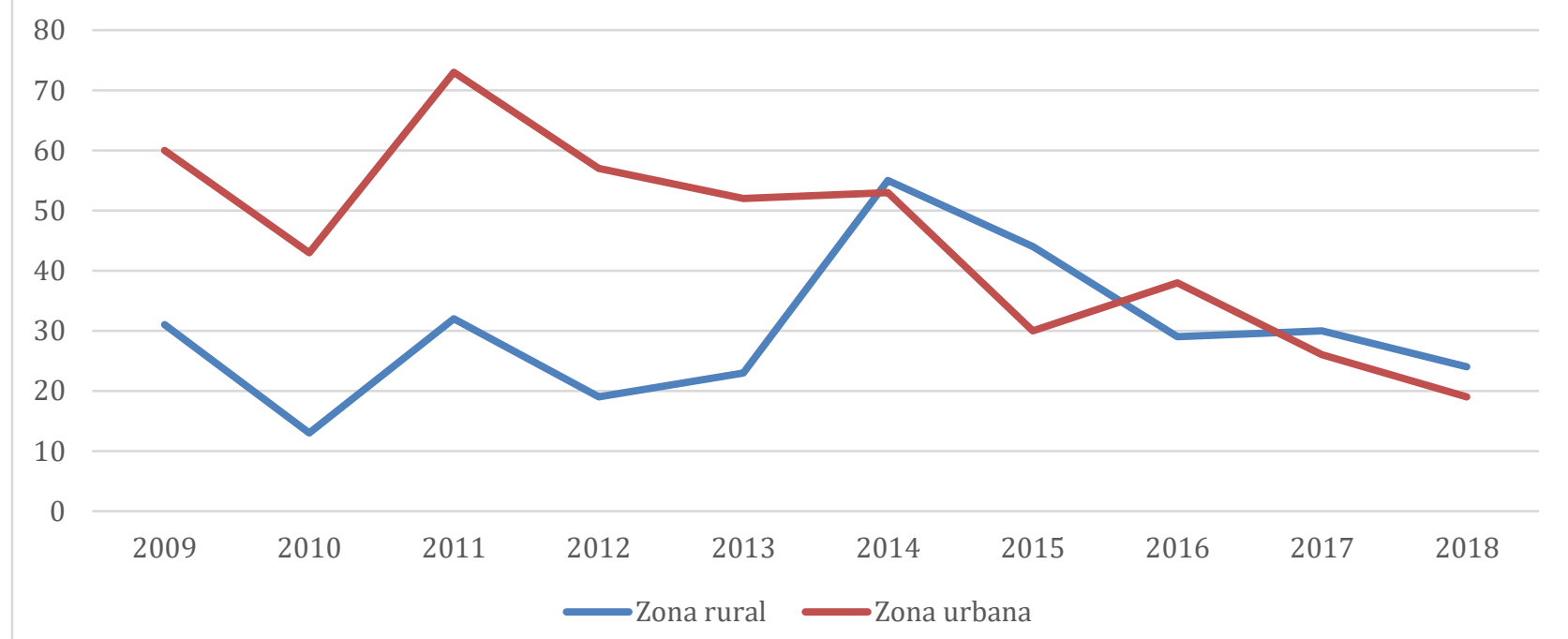

Fonte: Rios Junior WO, et al., 2020. Dados extraídos do Sistema de Informação de Agravos de Notificação (SINAN).

A maior concentração de casos de Leishmaniose Visceral na zona urbana também foi encontrada nos estudos realizados no Piauí (BATISTA FMA, et al., 2014). Isso se dá em razão de alguns fatores, dentre eles: na zona urbana, principalmente em cidades nordestinas, pode-se notar precariedade no saneamento básico, reflexo da baixa condição socioeconômica associada. 
Além disso, o desmatamento desenfreado, associado ao crescimento das cidades, destrói o habitat do flebotomíneo, vetor da Leishmaniose, causando sua migração para a zona urbana. Já a mudança do aspecto epidemiológico, observada no Gráfico 2, se deveu a uma redução da incidência na zona urbana, que pode estar relacionada a uma maior informação da população à cerca da prevenção da doença, bem como um aumento na incidência na zona rural, que pode estar relacionada a uma melhoria no processo de diagnóstico e notificação.

Considerando o grau de escolaridade da população atingida pela Leishmaniose visceral, observa-se no primeiro quinquênio que, dentre os 279 casos em que essa classificação foi descrita, 4,04\% dos pacientes eram analfabetos e $64,6 \%$ possuíam apenas o Ensino Fundamental Incompleto (EFI). Além disso, 5,05\% dos pacientes tinham Ensino Fundamental Completo (EFC), 10,01\% Ensino Médio Incompleto (EMI) e 14,1\% concluíram o Ensino Médio (EMC). Apenas 1 paciente (0,24\%) cursava o Ensino Superior (ESI) e 1 já o havia concluído (ESC).

O predomínio da escolaridade EFI também foi visualizado no segundo período analisado no estudo, com $75,27 \%$ dos casos. Além disso, 6,45\% eram analfabetos, mesmo percentual de indivíduos com EFC. Por fim, 8,6\% não concluíram o Ensino Médio, enquanto 3,22\% o concluíram. Nenhum paciente cursou Ensino Superior. Observa-se, portanto, que não houveram mudanças significativas da escolaridade predominante dos indivíduos infectados, durante os últimos anos. A baixa escolaridade dos indivíduos acometidos também foi encontrada em estudos realizados em Rio Grande do Norte, em Paracatu-MG e no Ceará (BARBOSA IR, 2013; OLIVEIRA EM e PIMENTA AM, 2018; CAVALCANTE IJM e VALE MR, 2014). Resultados semelhantes foram encontrados no município de Sobral de 2001 a 2010 (OLIVEIRA LS, et al., 2013).

A relação entre a baixa escolaridade e uma maior incidência da doença pode ocorrer em razão do limitado acesso à informação, principalmente no que se refere às medidas de prevenção e controle do vetor da Leishmaniose Visceral. A baixa escolaridade está associada, também, a piores condições sociais, que refletem em um meio mais propício para o desenvolvimento da doença.

Conforme o resultado encontrado no estudo de Castro JM, et al. (2016), a falta de compreensão no mecanismo de transmissão da doença, geralmente fazendo uma associação a um hábito específico, sem qualquer esclarecimento aprofundado, resulta numa baixa crença na doença e, consequentemente, diminui a colaboração da população no processo.

Quanto à raça referida, a grande maioria dos indivíduos era parda, com $91,5 \%$ dos casos no primeiro quinquênio e 93,9\% no segundo. No Período 1, 22 (5,34\%) indivíduos eram de raça branca, 11 casos (2,67\%) de raça preta e apenas $2(0,48 \%)$ eram indígenas. Já no Período 2, $8(2,32 \%)$ indivíduos eram de raça branca, 13 casos $(3,78 \%)$ de raça preta e nenhum indígena foi acometido pela doença. Não houve, portanto, diferença significativa de incidência nessa variável, nos períodos analisados (Gráfico 3).

Gráfico 3 - Número de casos de Leishmaniose Visceral em Sobral, por raça, no período de 2009 a 2013 e de 2014 a 2018 (números absolutos).

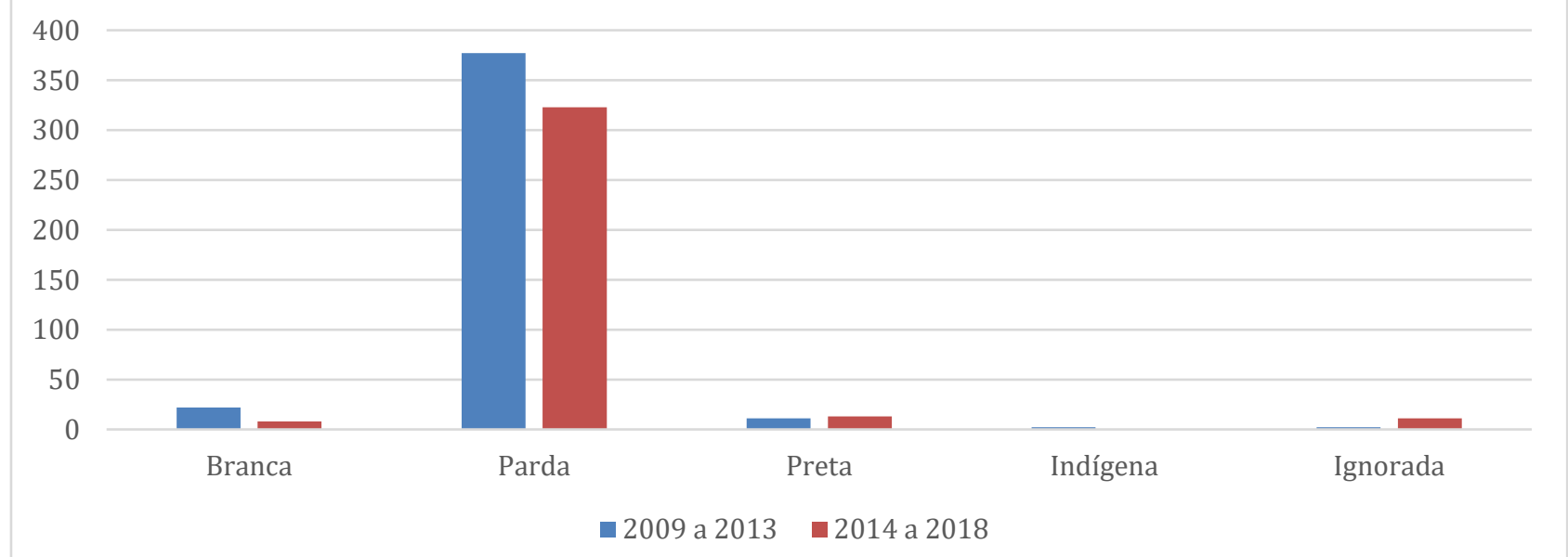

Fonte: Rios Junior WO, et al., 2020. Dados extraídos do Sistema de Informação de Agravos de Notificação (SINAN). 
Resultados semelhantes foram encontrados nos estudos realizados no Piauí e em Paracatu-MG (BATISTA FMA, et al., 2014; OLIVEIRA EM e PIMENTA AM, 2018). Também houve predomínio da doença em pardos, em estudo realizado no município na primeira década deste século (OLIVEIRA LS, et al., 2013).

Em relação a maior incidência da doença na população parda como não há relatos de estudos que determinem uma relação direta entre cor de pele e maior risco de desenvolver a doença, acredita-se que esse achado deve-se ao fato de que, na população de cor parda, concentram-se os indivíduos com menor escolaridade, bem como aqueles com menor nível socioeconômico, reflexo da desigualdade racial no país. Além disso, deve ser levado em consideração o percentual de pessoas autodeclaradas pardas no Ceará: mais de $60 \%$ dos residentes do estado (IBGE, 2020).

Com relação à distribuição do número de casos da doença por sexo, em Sobral, foi verificada uma incidência maior no sexo masculino em todos os anos do estudo. No primeiro período analisado, 261 homens (63\%) foram acometidos por Leishmaniose Visceral, percentual quase igual ao encontrado no segundo período, com $226(63,7 \%)$ indivíduos do sexo masculino contraindo a doença.

A maior proporção de casos no sexo masculino também foi encontrada no município na década passada e em estudos em outros locais do Brasil, como os realizados no Piauí, no Rio Grande do Norte e em ParacatuMG (OLIVEIRA LS, et al., 2013; BATISTA FMA, et al., 2014; BARBOSA IR, 2013; OLIVEIRA EM e PIMENTA AM, 2018). No estado do Ceará, como um todo, a incidência também é maior nos homens (CAVALCANTE IJM, VALE MR, 2014).

A maior incidência de Leishmaniose Visceral no sexo masculino não indica uma maior predisposição desse sexo ao desenvolvimento da doença. A maior incidência nos homens pode estar relacionada à maior exposição desse grupo ao flebótomo infectado, em razão de uma maior presença destes em locais extradomiciliares de risco, no exercício de suas atividades, principalmente em regiões de habitat desse vetor. Ao analisar a faixa etária, é possível dividir a amostra em três grupos: crianças/adolescentes (até 19 anos), adultos ( $>19$ anos) e idosos ( $\geq 60$ anos).

Nesse contexto, o grupo mais acometido pela doença foi o de crianças/adolescentes representando 58,45 $\%$ da amostra no primeiro quinquênio e $63,38 \%$ no segundo. No Período $1,41,5 \%$ dos acometidos eram adultos sendo 4,59\% idosos, enquanto no Período 2, 36,6\% eram adultos, sendo 5,91\% idosos.

A faixa etária mais acometida foi a de 1 a 4 anos com $24,64 \%$ dos casos no Período 1 e $30,42 \%$ no Período 2. Já entres adultos, o maior número de doentes tinha entre 20 e 39 anos com $23,2 \%$ das notificações no primeiro quinquênio e $18,9 \%$ no segundo (Gráfico 4).

Gráfico 4 - Número de casos de Leishmaniose Visceral em Sobral, por faixa etária, no período de 2009 a 2013 e de 2014 a 2018 (números absolutos).

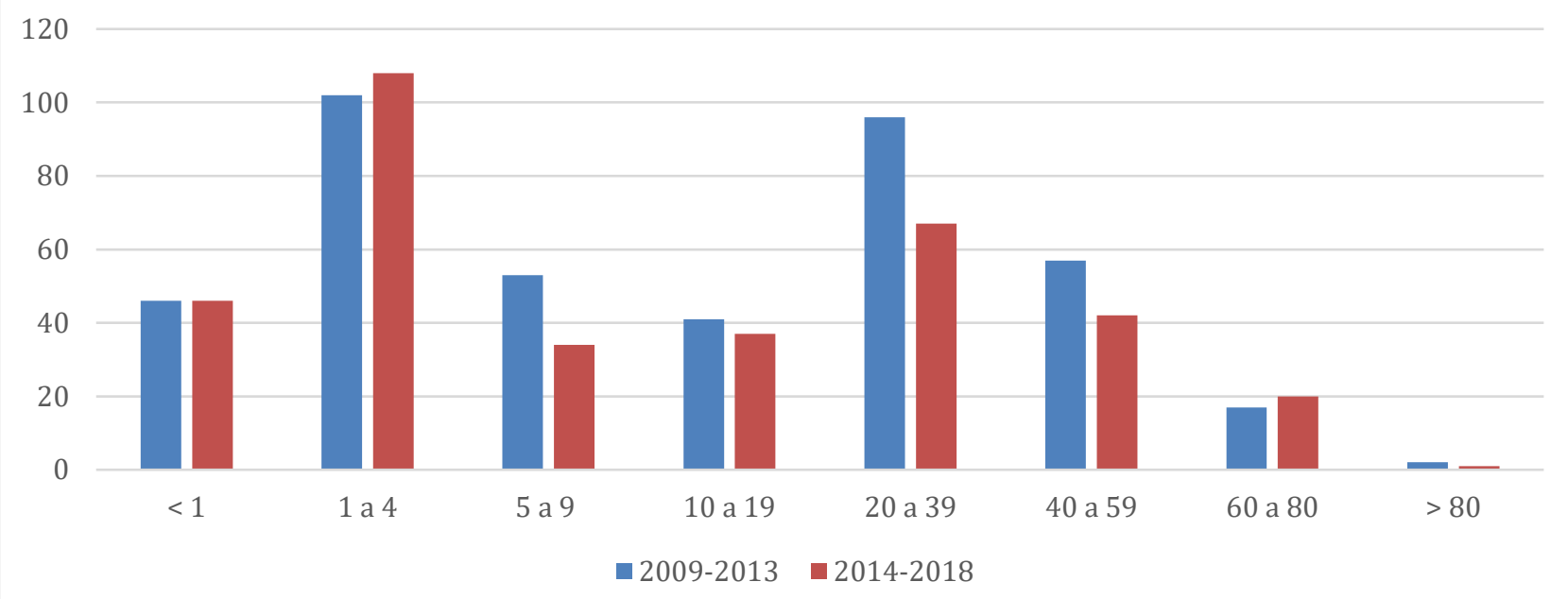

Fonte: Rios Junior WO, et al., 2020. Dados extraídos do Sistema de Informação de Agravos de Notificação (SINAN). 
Em relação ao maior número de casos em crianças, foram encontrados resultados semelhantes em estudos realizados em Natal e em Minas Gerais (BARBOSA IR, 2016; SILVA TAMS, et al.,2017). A faixa etária de 1 a 4 anos também foi a predominante em estudo realizado no estado (CAVALCANTE IJM, VALE MR, 2014).

Em relação às gestantes acometidas por Leishmaniose Visceral, apenas 1 caso foi notificado no primeiro quinquênio e apenas 3 casos no segundo, correspondendo a 0,24 e $0,84 \%$ do total de casos, respectivamente.

O aumento no número de casos, embora com reduzido número absoluto, representa um acréscimo de $200 \%$ na incidência de LV em gestantes, o que reforça a necessidade de orientação sobre a prevenção de contato com o vetor durante o acompanhamento pré-natal.

Ao analisar a coinfecção pelo vírus HIV, de 2009 a 2014, o número de casos positivos foi de 13 (3,14\%), enquanto houve uma queda no período de 2015 a 2018, com um total de 7 casos, ou seja, $1,97 \%$ da amostra. A média anual de casos foi de 2,6 $\pm 1,67$ no primeiro período e de 1,4 $\pm 1,34$ no segundo.

No que se refere a coinfecção pelo vírus HIV, carecem de estudos relacionados. O encontro da Leishmaniose Visceral e do vírus HIV seria inevitável, uma vez que a Leishmaniose vem apresentando um processo de urbanização e o HIV por uma interiorização (GOES MAO, et al., 2014).

A coinfecção com pelo vírus HIV, torna o diagnóstico mais difícil, pela sobreposição dos sintomas e ocorrência de manifestações clínicas atípicas, além de resultar, em muitos casos, repostas desfavoráveis ao tratamento clínico, recidivas frequentes da doença e aumentar, de forma importante, a mortalidade (SOUZA EC, et al., 2020).

Quanto ao critério de confirmação do diagnóstico de Leishmaniose Visceral utilizado, a grande maioria dos casos foi laboratorial, 390 (94,2\%) no Período 1 e 331 (93,24\%) no Período 2. Os demais casos obtiveram diagnóstico clínico-epidemiológico.

Desses pacientes, apenas $156(37,68 \%)$ realizaram teste parasitológico e $66(15,94 \%)$ realizaram Imunofluorescência Indireta no primeiro período, com positividade de $79,49 \%$ e $68,18 \%$, respectivamente. Já no segundo período, um número ainda menor realizou esses exames: 58 (16,34\%), com positividade de $92,86 \%$ para o exame parasitológico e 17 (4,79\%), com positividade de 58,82\%.

O diagnóstico da LV pode ser realizado através de fatores clínico-epidemiológicos, quando os sinais e sintomas típicos são encontrados em uma região endêmica da doença; através de exame parasitológico, por meio de um método invasivo (punção de órgãos); através do teste molecular, por técnica de Reação em Cadeia de Polimerase (PCR); através de testes imuno-histoquímico, ao colocar uma amostra em contato com anticorpos específicos; ou, por fim, através do método sorológico, ao observar a formação do anticorpo no soro do paciente pela técnica de ELISA (DOURADO ZF, et al., 2007).

Houve, portanto, uma queda na utilização do teste parasitológico e de Imunofluorescência Indireta para o diagnóstico da doença, embora haja predomínio do diagnóstico laboratorial nos períodos analisados. Tal fato pode ser explicado pelo custo elevado para realização desses testes, bem como a dificuldade na obtenção de material para o teste parasitológico, realizada através do aspirado de medula óssea.

Quando analisada a evolução clínica dos pacientes com leishmaniose visceral, no Período 1, após excluir os 26 casos em que a evolução foi ignorada, $63 \%$ dos doentes progrediram com a cura da doença e $6,4 \%$ evoluíram com óbito. $30,67 \%$ pacientes foram transferidos para outros serviços, sem ser possível identificar o desfecho nesses casos. Apenas $1(0,26 \%)$ caso foi contabilizado como abandono do tratamento. Já no Período 2, 60\% evoluiu para cura e 6,4\% para o óbito. 32,9\% dos pacientes foram transferidos para outro serviço e apenas $2(0,67 \%)$ abandonaram o tratamento.

O Coeficiente de Letalidade no Período 1 foi de $6,04 \%$ e a taxa de mortalidade média anual foi de 2,63 \pm 1,85 óbitos/100 mil habitantes. Já no Período 2 , o coeficiente de Letalidade foi de $5,35 \%$ e a taxa de mortalidade média anual foi de 1,88 $\pm 1,78$ óbitos/100 mil habitantes. $O$ ano com maior taxa de mortalidade foi 2011, que atingiu o número de 5,77 óbitos por 100 mil habitantes (Gráfico 5). 
Gráfico 5 - Taxa de mortalidade por Leishmaniose Visceral em Sobral no período de 2009 a 2018 (em óbitos por 100 mil habitantes).

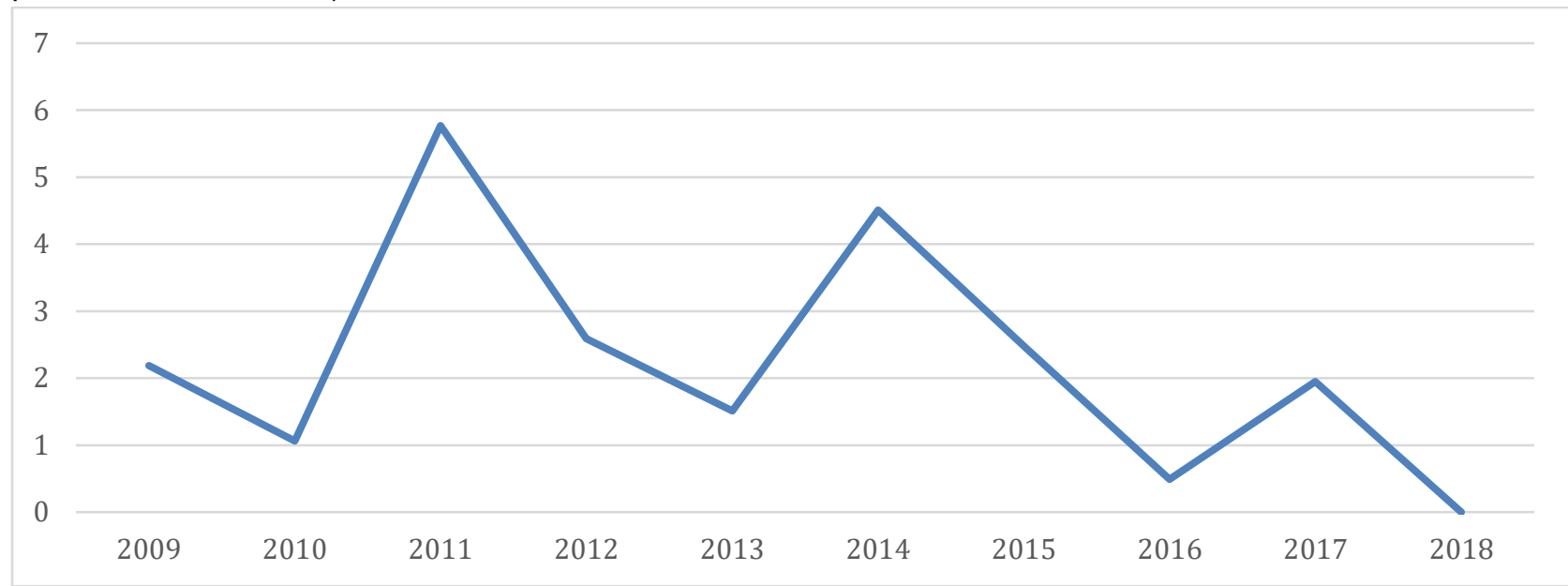

Fonte: Rios Junior WO, et al., 2020. Dados extraídos do Sistema de Informação de Agravos de Notificação (SINAN).

Embora haja uma variabilidade elevada no número de óbitos no decorrer dos anos analisados, observase uma queda de $28,52 \%$ na taxa de mortalidade da doença, ao realizar uma comparação entre os dois períodos do estudo. Além disso, houve uma redução em 11,42\% no Coeficiente de Letalidade. Tais resultados refletem uma melhora do sistema de saúde do município, no que se refere ao diagnóstico precoce e tratamento adequado e oportuno da doença.

\section{CONCLUSÃO}

O estudo evidenciou uma importante queda na incidência da Leishmaniose Visceral no município, bem como uma redução da sua taxa de mortalidade e de letalidade, fatores que refletem uma melhora do sistema de saúde, da prevenção ao diagnóstico e tratamento. Entre os períodos analisados, o predomínio da doença em indivíduos do sexo masculino, de faixa etária pediátrica, pardos e com baixo nível de escolaridade, se manteve. Foi possível observar, ainda, uma inversão no fenômeno de urbanização da doença, que passou a não possuir zona de residência predominante. Ademais, os resultados são uteis para compreensão da distribuição e comportamento da Leishmaniose Visceral em Sobral, possibilitando reforço nas estratégias de saúde no combate à doença.

\section{REFERÊNCIAS}

1. BARBOSA, IR. Epidemiologia da Leishmaniose Visceral no estado do Rio Grande do Norte, Brasil. Rev Epidemiol Control Infect. 2013;3(1):17-21.

2. BARBOSA IR. Leishmaniose Visceral Humana no Município de Natal-RN: Análise clínica-epidemiológica e espacial. Ver Ciênc Plur. 2016; 2(1):89-101.

3. BATISTA FMA, et al. Leishmaniose: perfil epidemiológico dos casos notificados no estado do Piauí entre 2007 e 2011. Rev UNIVAP, 2014; 20 (35): 44-55.

4. CASTRO JM, et al. Conhecimento, Percepções de Indivíduos em Relação à Leishmaniose Visceral Humana Como Novas Ferramentas de Controle. Ensaios Cienc., Cienc. Biol. Agrar. Saúde, 2016; 20 (2): 93-103

5. CAVALCANTE IJM, VALE MR. Aspectos epidemiológicos da leishmaniose visceral (calazar) no Ceará no período de 2007 a 2011. Rev Bras Epidemiol. 2014; 17(4): 911-924.

6. CEARÁ. Boletim epidemiológico leishmaniose visceral [online]. Secretaria da Saúde do Estado do Ceará, 2018. Disponível em: $<$ https://www.saude.ce.gov.br/wpcontent/uploads/sites/9/2018/06/boletim_leishimaniose_24_10_2018.pdf>. Acesso em: 28 ago. 2020.

7. COSTA DNCC, et al. Leishmaniose visceral em humanos e relação com medidas de controle vetorial e canino. Rev Saude Publica. 2018; 52 (92): 1-11.

8. DOURADO ZF, et al. Panorama histórico do diagnóstico laboratorial da leishmaniose visceral até o surgimento dos testes imunocromatográficos (RK39). Revista De Patologia Tropical / Journal of Tropical Pathology, 2007; 36(3): 205214. 
9. GÓES MAO, et al. Urbanização da leishmaniose visceral: aspectos clínicos e epidemiológicos em Aracaju, Sergipe, Brasil. Rev Bras Med Fam Comunidade. Rio de Janeiro, 2014; 9(31):119-126.

10. IBGE. Estimativas da População [online]. IBGE, 2020. Disponível em: https://www.ibge.gov.br/estatisticas/sociais/populacao/9103-estimativas-de-populacao.html. Acesso em: 28 ago. 2020.

11. LUCENA RV, MEDEIROS JS. Caracterização epidemiológica da leishmaniose visceral humana no nordeste brasileiro entre 2010 e 2017. Journal of Biology \& Pharmacy and Agricultural Management. 2018; 14 (4): $285-298$.

12. MINISTÉRIO DA SAÚDE. Leishmaniose Visceral: o que é, causas, sintomas, tratamento, diagnóstico e prevenção [online]. Ministério da Saúde, 2020. Disponível em: <http://www.saude.gov.br/saude-de-a-z/leishmaniosevisceral\#epidemiologia>. Acesso em 28 ago. 2020.

13. OLIVEIRA EM e PIMENTA AM. Perfil epidemiológico das pessoas portadoras de leishmaniose visceral no município de Paracatu - MG no período de 2007 a 2010. Rev Min Enferm. 2014; 18(2): 365-370.

14. OLIVEIRA LS, et al. Perfil epidemiológico dos casos de leishmaniose Visceral em sobral, Ceará no período de 2001 a 2010. S A N A R E, 2013; 12 (1):13-19.

15. ORGANIZAÇÃO PAN-AMERICANA DA SAÚDE. Leishmanioses: Informe Epidemiológico das Américas [online]. OPAS, 2019. Disponível em: <https://iris.paho.org/bitstream/handle/10665.2/50505/2019-cde-leish-informe-epi-dasamericas.pdf?sequence=2\&isAllowed $=y>$. Acesso em 28 ago. 2020.

16. RODRIGUES ACM, et al., Epidemiologia da leishmaniose visceral no município de Fortaleza, Ceará. Pesq. Vet. Bras. 2017; 37(10):1119-1124.

17. SILVA TAMS, et al. Spatial and temporal trends of visceral leishmaniasis by mesoregion in a southeastern state of Brazil, 2002-2013. PLoS Negl Trop Dis, 2017; 11 (10): 1-20.

18. SOUZA EC, et al. Apresentação clínica da leishmaniose visceral em pacientes portadores do HIV: Análise dos Fatores Relacionados ao Aparecimento da Doença. Braz. J. Hea. Rev., 2020; 3 (2):1766-1777.

19. WERNECK GL. Controle da leishmaniose visceral no Brasil: o fim de um ciclo? Cad. Saúde Pública, 2016 ; 32 (6): eED010616.

20. WORLD HEALTH ORGANIZATION. Leishmaniasis [online]. WHO, 2020. Disponível em: <https://www.who.int/en/news-room/fact-sheets/detail/leishmaniasis>. Acesso em 28 ago. 2020. 